\title{
Nuclear Phosphoproteomics Features the Novel Smoking Markers in Mouse Lung Tissue Following Subacute Phase Exposure to Tobacco Smoke
}

\author{
Kanako Niimori-Kita ${ }^{1 *}$, Fumiko Nakamura ${ }^{1}$, Daikai Koizumi ${ }^{1}$ and Daisuke Niimori ${ }^{* *}$ \\ ${ }^{1}$ Department of Pathology and Experimental Medicine, Kumamoto University, Honjo, Kumamoto, Japan \\ ${ }^{2}$ Department of Dermatology and Plastic Surgery, Graduate School of Life Sciences, Kumamoto University, Honjo, Kumamoto, Japan
}

\begin{abstract}
Smoking is a risk factor of lung diseases including chronic obstructive pulmonary disease (COPD) and lung cancer. However, the molecular mechanisms inducing these diseases remain to be completely uncovered. In order to elucidate them, it is necessary to identify the signaling pathway activated by tobacco smoking exposure. Especially, it is important to identify nuclear phosphoproteins induced by tobacco smoking exposure because the signaling pathways are modified by phosphoproteins. This time, to identify nuclear phosphoproteins as novel smoking markers, nuclear phosphoproteimics of mouse lung tissue following tobacco smoking exposure was examined. Tobacco smoking exposure against mice was examined using the nose-only, flow-past inhalation exposure chamber system for one month. Phosphopeptides eluted from nuclear proteins of the tobacco exposured mice lungs were identified by mass spectrometry. The result showed that 77 phosphoproteins were totally identified. Among them, the semiquantitative analysis using ProteolQ proteomic software revealed that five phosphoproteins showed the different expression patterns between control and tobacco exposure groups. Furthermore, the classification by biological functions of the identified proteins revealed that these proteins were related to inflammation, regeneration, repair, proliferation, differentiation, morphological change and nicotine or stress response. Finally, we founded advanced glycosylation end product-specific receptor (RAGE) and serine/threonine-protein kinase SNF1-like kinase 2 (SIK2) as novel smoking markers.
\end{abstract}

Keywords: Nuclear phosphoproteomics; Subacute phase; Tobacco smoke; Lung; RAGE; SIK2

\begin{abstract}
Abbreviations
COPD: Chronic Obstructive Pulmonary Disorder; RAGE: Advanced Glycosylation End Product-Specific Receptor; SIK2: Serine / Threonine-Protein Kinase; SNF1: Like Kinase; HE: Hematoxylin-Eosin; MS: Mass Spectrometry; TFA-A: Trifluoroacetic Acid-Acetonitrile; ESI-TRAP: Electrospray Ionization-Trap; DAPI, 4', 6-Diamino-2Phenylindole Dihydrochleride; EMT: Epithelial Mesenchyal Transition or Transformation; CAMK: Calmodulin-Dependent Protein Kinase; TORC: Transducer of Regulated CREB; CREB: cAMP Responsive Element-Binding Protein; PASMC: Pulmonary artery Smooth Muscle Cell
\end{abstract}

\section{Introduction}

Smoking is widely considered the risk factor of lung diseases including COPD [1-3], and lung cancer [4]. In order to elucidate the molecular mechanisms of these diseases, the various researches have been examined [5]. However, they remain to be completely identified. These diseases are induced by serious tobacco smoking exposure, and it shows that accumulation of cell injury by smoking causes diseases partly. Therefore, though there are many reports about the experiments of long term exposure of tobacco smoke [6,7], there are few reports about subacute smoking exposure [8,9]. The subacute smoking exposure against mice induces the activation of processes such as various signaling pathways, immune reaction $[10,11]$, oxidative stress $[12,13]$, spontaneous mutation of somatic cell [14-19] and epigenetic regulation $[12,20]$. These phenomena also apply to human.

To elucidate the molecular mechanisms of smoking exposure, the experimental approaches focusing the toxic changes by diseases, such as cell proliferation [21], chronic inflammatory [22,23] and apotosis [24] have been examined. On the other hand, we need novel biomarkers of diseases induced by toxic substances exposure in the environment because biomarkers give effective tools for the early diagnosis, prevention, and cure of these diseases. Smoking marker genes have been screened using cDNA microarray or microRNA analysis $[25,26]$. However, such information is not enough for elucidating the mechanisms. For instance, cDNA microarray cannot screen the functional changes including post-translational modification such as phosphorylation. However, we can find the molecules playing an important role in vivo using proteomics because it is an effective tool for screening post-translational modification, proteins, signaling pathway controlling biological events. Proteomics gives us an understanding of lung diseases by smoking exposure and signaling pathway plays a role in estimating biological changes by human diseases. However, the changes of signaling pathway in response to the toxic substances have not been uncovered yet. Therefore, it is necessary to construct the best method for detecting post-translational modification such as phosphorylation, proteins changes in response to signaling in human and animal tissues.

${ }^{*}$ Corresponding authors: Kanako Niimori-Kita, Department of Pathology and Experimental Medicine Kumamoto University, 1-1-1, Honjo, Kumamoto 860-8556, Japan, Tel: +81-96-373-5086; Fax: +81-96-373-5087; E-mail: kitakana@kumamoto-u.ac.jp

Daisuke Niimori, Department of Dermatology and Plastic Surgery, Kumamoto University, 1-1-1, Honjo, Kumamoto 860-8556, Japan, Tel: +81-96-373-5233; Fax: +81-96-373-5235; E-mail: nimosuke7@yahoo.co.jp

Received February 19, 2016; Accepted February 27, 2016; Published March 05, 2016

Citation: Niimori-Kita K, Nakamura F, Koizumi D, Niimori D (2016) Nuclear Phosphoproteomics Features the Novel Smoking Markers in Mouse Lung Tissue Following Subacute Phase Exposure to Tobacco Smoke. J Bioanal Biomed 8: 009016. doi:10.4172/1948-593X.1000146

Copyright: (c) 2016 Niimori-Kita K, et al. This is an open-access article distributed under the terms of the Creative Commons Attribution License, which permits unrestricted use, distribution, and reproduction in any medium, provided the original author and source are credited. 
In previous study, we identified nuclear phosphoproteins as novel tobacco markers in mouse lung tissue following short-term exposure to tobacco smoke by original proteomic strategy [27]. It is considered that markers control the molecules functioning such as biological events inflammation, repair, proliferation and differentiation. According to this method, we tried to detect nuclear phosphoproteins in mice lung tissues following one month exposure to tobacco smoke corresponding to the late subacute phase. Using the nose-only, flow-past inhalation exposure chamber system, mainstream smoking exposure against mice was examined for one month [28].

Phosphopeptides eluted from nuclear proteins of the tobacco exposured mice lungs were identified by mass spectrometry. Phosphoproteins of 45 (Control specific group; 12, Tobacco specific group 25; both groups 8 ) were identified. Moreover, to find proteins whose expression level changes in response to tobacco smoking exposure, semi-quantitative analyses were examined. Finally, then we analyzed RAGE and SIK2 as novel smoking markers.

\section{Materials and Methods}

\section{Animals and tobacco smoke exposure}

Conditions: A total of 16 male ICR mice weighting $45-50 \mathrm{~g}$ and aged 10 weeks were used. The mice were obtained from Japan SLC, Inc (Shizuoka, Japan), and were housed in a room illuminated for 12 $\mathrm{h}$ (lights on 07:00 - 19:00) and kept at $22 \pm 2^{\circ} \mathrm{C}$ during the experiment. Food (CE2, CLEA Japan, Inc., Tokyo, Japan) and water were freely available. The mice were divided into two groups; one month control and one month exposed $(n=8$, each).

To expose the mice to tobacco smoke, nose only flow post inhalation exposure chamber system was used. The system consists of three parts, tobacco smoke generator (SCIREQ Inc., Montreal, Canada), exposure chamber (CR Equipment S.A., Gland, Switzerland), and air filtering system and compressor (NES-1000, SHINTECHNO, Fukuoka, Japan). Compared to conventional systems, it suppresses the effect of secondhand smoke (Figure 1). The exposure experiment was conducted in an iteration process; tobacco smoke is puffed in the mice's muffle and the mice aspire the smoke, and then the exhaled smoke (and the rest of the smoke) is removed by the vacuum system. The mice were exposed to tobacco smoke for one month, in a chamber for $30 \mathrm{~min}$ once a day. 24 cigarettes of University Kentucky 3R4F, cigarettes for animal tobacco exposure experiment, were burned in a day experiment and each cigarette was puffed 10 times. Control mice were held in the mouse holders in the same way and exposed to the room air for the same duration of the exposed groups [27].

Sample preparation: For morphological study, 4 mice in each group were sacrificed under intra-abdominal pentobarbital, and the lung tissues were isolated and fixed with phosphate-buffered $4 \%$ paraformaldeyde. The tissues were embedded in paraffin and the sections were stained with hematoxylin and eosin (HE) stains.

For proteomic study, 4 mice in each group were sacrificed within 1 $\mathrm{h}$ after the last exposure. The right lung was used for protein extraction. After perfusion with PBS including protease and phosphatase inhibitors, lung tissue was isolated. To enrich nuclear protein, lung tissue was homogenized on ice in the elution buffer of nuclear protein dissociation kit (Thermo Scientific Pierce, Rockford, IL, USA). The mixture was vortexed for $15 \mathrm{sec}$ followed by $10 \mathrm{~min}$ incubation on ice. After adding cytosolic elution buffer, the mixture was vortexed for 5 $\mathrm{sec}$, followed by 1 minute incubation on ice. It was centrifuged at 15,000 $\mathrm{rpm}$ for $5 \mathrm{~min}$ and the supernatant was removed as cytosolic protein extract. The pellet was vortexed in nuclear dissociation buffer for 15 sec every $10 \mathrm{~min}$, which was performed for $40 \mathrm{~min}$. The extract was centrifuged at $15,000 \mathrm{rpm}$ for $10 \mathrm{~min}$, and the supernatant including nuclear proteins was harvested. The nuclear proteins were digested by trypsin for peptide elution. And to isolate phosphopeptides selectively, polymer-based metal ion affinity capture (PolyMAC, Tymora Analytical Operations, LLC, West Lafayette, IN, USA) was used. The nuclear peptide extracts were reacted with $\mathrm{TiO}_{2}$ coated-magnetic capture beads for catching phosphopeptides [29]. After being agitated, nuclear phosphopeptides were eluted. The samples were resolved into TFA-A (0.1\% TFA / $2 \% \mathrm{MeCN} / \mathrm{DW})$, and analyzed by ESI-TRAP [27].

Mass spectrometry: The samples were analyzed by mass spectrometry. The $\mu$-HPLC/NSI-MS/MS system was composed of Advance Nano UHPLC(Michrom Bioresources Inc., Auburn, CA, USA) for HPLC, HTS-PAL auto sampler (CTC Analysis, Zwingen, Switzerland), and mass spectrometer (amaZonTM ETD, Bruker, Billerica, MA, USA). The samples were loaded into a reversed phase column, Zaplous column C183 m $0.1 \mathrm{~mm} \mathrm{lD} \times 150 \mathrm{~mm}$ (AMR Inc., Tokyo, Japan) for separation, and the nuclear phosphoproteins were identified [27].

Data Analysis All MS/MS data were investigated using the MASCOT search engine (Matrix Science, Ltd., London, UK) against the Swiss-Prot database. The data obtained from 4-protein digests were investigated against the other mammalian subsets of the sequences. The MS/MS data were searched through the mus musclus (mouse) subsets of the sequences. The database search was performed allowing for fixed modification on cysteine residue (carbamidomethyl, + 57 $\mathrm{Da}$ ), variable modification on methionine residue (oxidation, +16 $\mathrm{Da}$ ), serine/threonine residue (phosphorylation), and tyrosine residue (phosphorylation), peptide tolerance at $\pm 2.0 \mathrm{Da}$, and fragment mass tolerance at $\pm 0.8 \mathrm{Da}[27]$.

Immunostaining: Among the candidate nuclear phosphoproteins identified by mass spectrometry, we focused on RAGE and SIK2, and performed the immunofluorescent staining for these molecules in order to confirm the expression pattern and localization. Control and tobacco

A

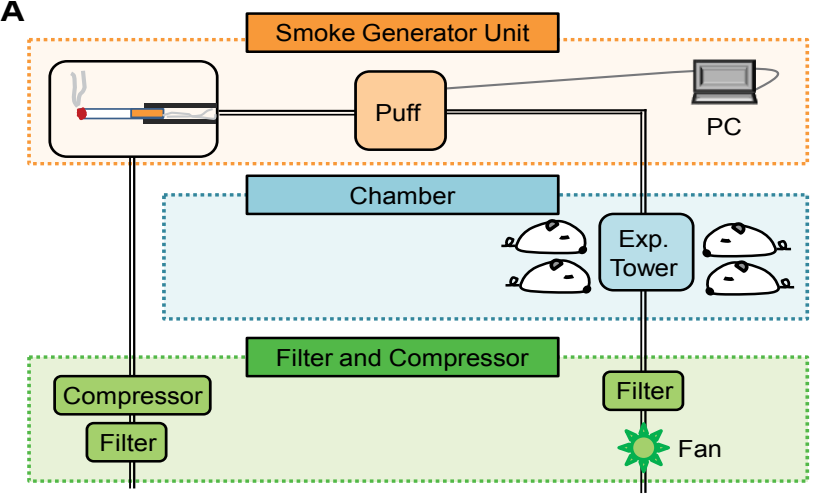

B
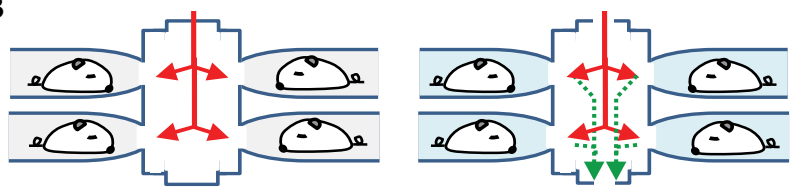

Figure 1: Panel A: Schematic of the nose-only, flow-past inhalation exposure chamber system. Panel B: Schematic of the tobacco exposure system. This system can remove the exhaled smoke and suppresses the effect of secondhand smoke (right). 
exposure lung tissues were embedded with OCT compound. Frozen section's size was $10 \mu \mathrm{m}$. After activation, lung tissue sections were blocked with $1 \%$ bovine serum albumin for $30 \mathrm{~min}$, and were washed 3 times with PBS. Then, they were reacted with primary antibodies diluted at 1:1000 for 2 hours. After washing with PBS 3 times, they were also reacted with secondary antibodies diluted at 1:100 for 1 hour. Immunoreactive signals were detected using Alexa488 fluorescent dye of tyramide signal amplification kit (T20922, Life technologies, USA, OR). 4', 6-Diamino-2- Phenylindole, Dihydrochleride (DAPI) was diluted with the mounting agent. Using this DAPI containing mounting agent, the lung tissues were enclosed. The immunoreactive fluorescent signals were observed using an epifluorescent microscope system (OLYMPUS, Tokyo, JP). An anti-RAGE antibody (ab3611, Abcam, Milton, Cambridge, UK and an anti-SIK2 antibody (LS-B2068, LifeSpan Biosciences Inc., Seattle, WA, USA) were used as primary antibodies in this study.

\section{Results}

Histological changes induced by tobacco exposure We examined the tobacco exposure experiment for one month using the nose only flow post inhalation exposure chamber system in which mice are exposed to the mainstream smoke (Figure 1). In order to verify that the tissues were modified after exposure to tobacco smoke for one month, we observed HE-stained lung tissues (Figure 2). The results showed thickening of the alveolar wall and accumulation of inflammatory cells (Figure 2).

Biological functional analyses of identified nuclear phosphoproteins In order to identify nuclear phosphoproteins changed by tobacco smoking exposure, we collected phosphopeptides eluted from nuclear proteins of the tobacco exposured mice lungs, and identified phosphoproteins by mass spectrometry such as DNA topoisomerase 2-beta (Figure 3). We examined the mass spectrometry analyses two times for repeatability.

A total of 45 identified nuclear phosphoproteins included specific proteins and common proteins in control and tobacco groups. The number of commonly expressed proteins in both groups was 8 . Additionally, 12 proteins were identified as control specific proteins, while 25 proteins were identified as tobacco specific proteins (Figure 4). Next, the identified phosphoproteins were analyzed semi-quantitatively in silico by ProteoIQ software, and a heat map of the proteins was constructed to show the differential expression of proteins in each group. A total of 45 proteins showed significantly different expression between control and tobacco groups (Figure 5). Not only the proteins changed by tobacco exposure such as RAGE in previous report but also the novel proteins, SIK2, were identified this time (Figure 5).

Identified phosphoproteins were classified according to biological function by ProteoIQ software, and included proteins were related to Epidermal-Mesenchymal transition or transformation (EMT),

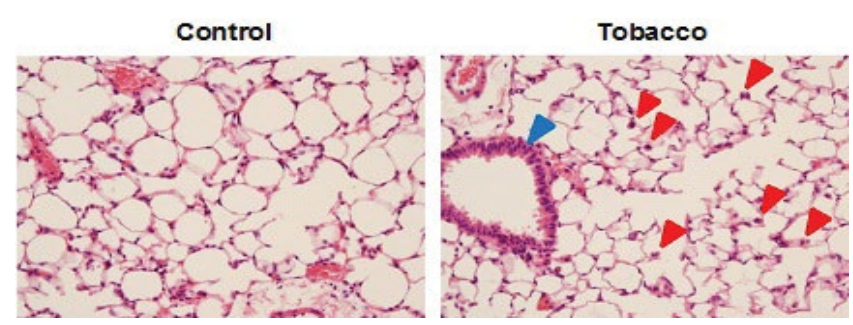

Figure 2: Micrographs of lung tissues obtained from control mice and mice exposed to tobacco smoke for one month. inflammation, tobacco response, regeneration, proliferation, morphogenesis, apoptosis, regulation and complex. Especially, the expression of proteins constructing the complex was decreased by one month smoking exposure (Figure 6). On the other hand, the expression of proteins functioning as process, regulation, response and activity was up-regulated by one month smoking exposure (Figure 6). Among them, we focused on SIK2 and RAGE showing differential expression pattern in response to the smoking exposure, and examined functional analyses such as Immunostaining for the proteins. Changing of SIK2 and RAGE in response to smoking exposure.

We examined the Immunostaining for SIK2 in the lung tissue (Figure 7A). In the control group, SIK2 was expressed in the cytoplasm of the bronchial tubes, and also highly expressed in the cytoplasm and nucleus of alveolus (Figure 7A). On the other hand, the expression of SIK2 in the cytoplasm and nucleus was decreased in the tobacco group compared to the control group (Figure 7A). These results indicated that the SIK2 expression in the cytoplasm and nucleus of the lung tissue was decreased by the smoking exposure.

Next we focused on RAGE as a candidate smoking marker because it was expressed in response to the smoking exposure. In the previous reports, western blot analyses showed that RAGE is induced by inflammation in response to the smoking exposure [30,31]. Moreover, it is also reported that RAGE is usually expressed in cytoplasm of the alveolar type I epithelial cell and the alveolar macrophage [32]. However, there is no report showing the Immunostaining of the lung tissue. Therefore, we examined the Immunostaining in order to confirm the localization of RAGE in the lung tissue (Figure 8). The Immunostaining results revealed that RAGE was expressed in not only the cytoplasm but also the nucleus of the alveolar type I and epithelial cell and the alveolar macrophage (Figure 8). These results indicated that a part of RAGE entered into the nucleus in response to the smoking exposure. Moreover, this demonstrates that nuclear proteins were approximately purified using our methods.

\section{Discussion}

The swelling of alveolar epithelial cell, the thickening and the partial destruction of alveolar epithelial wall, the expansion of airspace and the infiltration of alveolar macrophage were induced by the sub-acute term tobacco smoking exposure such as 1 month tobacco smoking exposure [33-35]. This time, we observed the thickening of alveolar epithelial wall and the infiltration of alveolar macrophage. These morphological changes were induced by the activation or inactivation of molecules in multiple signaling pathways. For instance, the transcription factors are activated or inactivated by the change of signaling pathway. The transcription factors are phosphorylated or dephosphorylated in the cytoplasm, and enter into the nucleus, and they control the downstream proteins. This indicates that the changes of nuclear phosphoproteins reflect the fluctuation of multiple signaling pathways. Proteomic analyses are useful for detecting them. In previous, in order to identify the tobacco smoking marker in the lung tissue, proteomic analyses was examined [36]. Our study also searches for the nuclear phosphoproteins changed in the mice lung by the tobacco exposure using the original proteomic strategy.

However, the proteomic analyses of whole proteins in the tissue are not enough for detecting the signaling pathway changed in response to tobacco exposure. Because the amount of nuclear phosphoproteins is very low, the detection of whole proteins including them slightly is difficult. Then, we attempted to elute nuclear phosphoproteins by concentrating the phosphopeptides after the nuclear protein elution 
Citation: Niimori-Kita K, Nakamura F, Koizumi D, Niimori D (2016) Nuclear Phosphoproteomics Features the Novel Smoking Markers in Mouse Lung Tissue Following Subacute Phase Exposure to Tobacco Smoke. J Bioanal Biomed 8: 009-016. doi:10.4172/1948-593X.1000146

A
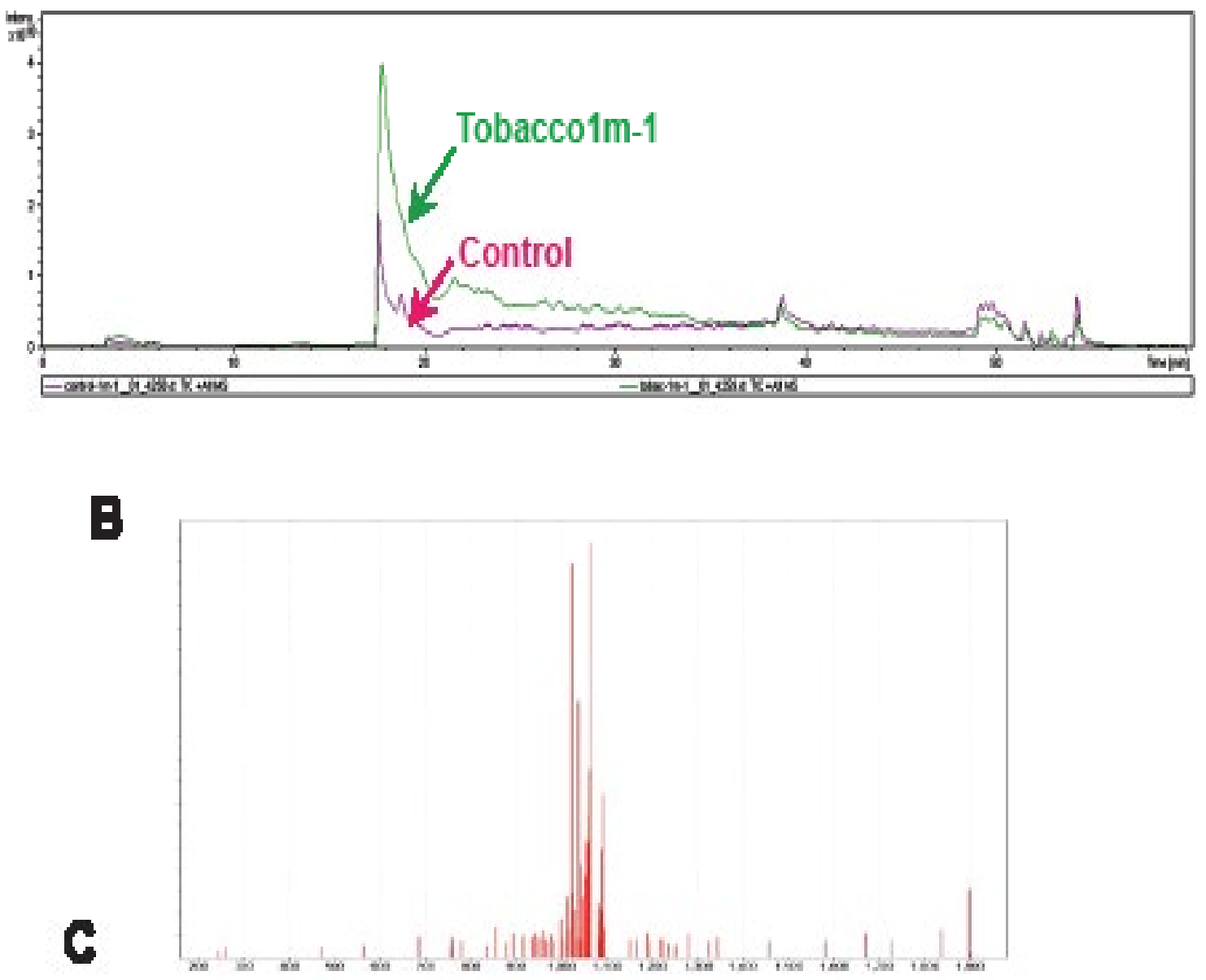

\begin{tabular}{|c|c|c|c|c|}
\hline$\#$ & b & Sequence & $y$ & $\#$ \\
\hline 1 & 129.102795 & $\mathrm{~K}$ & 2138.930397 & 18 \\
\hline 2 & 242.186865 & I & 2010.835427 & 17 \\
\hline 3 & 341.255285 & V & 1897.751357 & 16 \\
\hline 4 & 470.297885 & $E$ & 1798.682937 & 15 \\
\hline 5 & 571.345565 & $\mathrm{~T}$ & 1669.640337 & 14 \\
\hline 6 & 684.429635 & I & 1568.592657 & 13 \\
\hline 7 & 798.472565 & $\mathrm{~N}$ & 1455.508587 & 12 \\
\hline 8 & 965.470926 & $S+79.9663$ & 1341.465657 & 11 \\
\hline 9 & 1080.497876 & D & 1174.467296 & 10 \\
\hline 10 & 1247.496237 & $S+79.9663$ & 1059.440346 & 9 \\
\hline 11 & 1362.523187 & D & 892.441985 & 8 \\
\hline 12 & 1449.555217 & $S$ & 777.415035 & 7 \\
\hline 13 & 1578.597817 & $E$ & 690.383005 & 6 \\
\hline 14 & 1725.666237 & $F$ & 561.340405 & 5 \\
\hline 15 & 1782.687707 & G & 414.271985 & 4 \\
\hline 16 & 1895.771777 & I & 357.250515 & 3 \\
\hline 17 & 1992.824547 & $\mathrm{P}$ & 244.166445 & 2 \\
\hline 18 & 2120.919517 & $\mathrm{~K}$ & 147.113675 & 1 \\
\hline
\end{tabular}

Figure 3: Panel A: Spectrum of the detected peptides (control group in pink and tobacco exposed group in green). Pink arrows indicate control-specific peaks and green arrows indicate tobacco exposure-specific peaks. The vertical axis indicates intensity, and the horizontal axis indicates retention time, which has a linear relationship with the molecular mass of the protein. MS/MS data of DNA topoisomerase 2-beta peptide as a representative phosphoprotein detected in this study. Panel B: show the MS/MS spectra used to identify the m/z 2120.91 ion-trap peak as a fragment of DNA topoisomerase 2- beta peptide KIVETINpSDPSDSEFGIPK. Panel C: Fragment ion table of KIVETINpSDpSDSEFGIPK. Peptide fragment ions of which charge is retained on the N-terminus are indicated by " $b$ ", and of which charge is maintained on the C-terminus are indicated by " $y$ ". "\#" is the number of peptide fragments. 


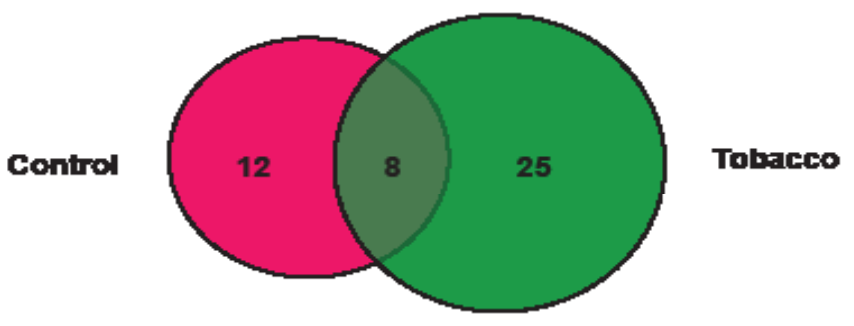

Figure 4: Venn diagram showing the identified protein profile overlaps between control and tobacco group. The area of each circle is proportional to the number of identified proteins.

\begin{tabular}{|c|c|c|c|}
\hline No. & Identified Proteins & Control-1M & Tobacco-1M \\
\hline 1 & Serine / threonine-protein kinase (SIK2) & 2 & 0 \\
\hline 2 & guanine nucleotide binding protein, alpha inhibiting 1 (GNAI1) & 1 & 0 \\
\hline 4 & Advanced glycosilation end product-specific receptor (RAGE) & 0 & 1 \\
\hline 5 & DNA topoisomerase 2-beta & 0 & 2 \\
\hline 6 & acidic ribosomal phosphoprotein $\mathrm{P} 1$ isoform 1 & 0 & 6 \\
\hline 7 & acidic ribosomal phosphoprotein P1 isoform 2 & 0 & 6 \\
\hline 8 & catenin alpha-1 & 0 & 1 \\
\hline 9 & myosin-1 & 0 & 1 \\
\hline 10 & serum deprivation-response protein & 0 & 3 \\
\hline 11 & myosin heavy chain Ila & 0 & 1 \\
\hline 12 & $60 S$ acidic ribosomal protein P2 & 0 & 5 \\
\hline 13 & brother of CDO precursor & 0 & 6 \\
\hline 14 & $60 S$ acidic ribosomal protein $\mathrm{P} 1$ & 0 & 6 \\
\hline 15 & myosin-8 & 0 & 1 \\
\hline
\end{tabular}

Figure 5: Heat map of phosphoproteins exhibiting differential expression after exposure to tobacco smoke. The table shows the result of semi-quantitative analysis using ProteolQ software. "Identified phosphoproteins" represents the list of protein names detected by MS. The number in each cell indicates the number of phosphopeptides detected by MS.
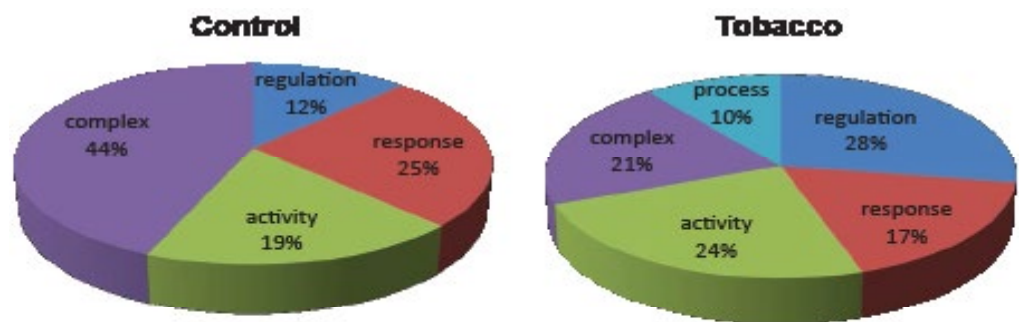

Figure 6: Pie charts representing the biological functional classification of identified proteins in each group. Identified proteins were classified by ProteolQ software. The area of each circle is proportional to the number of proteins identified in each group.

and trypsin digestion. As a result, we could identify the tobacco smoking candidate marker proteins such as RAGE and SIK2. In previous, RAGE and HSP were increased by one month tobacco exposure [37]. It was confirmed that RAGE was increased in the alveolar epithelial cells by tobacco exposure [36,38-41]. This time, RAGE was increased in response to tobacco exposure, and localized in the nucleus. RAGE promotes PASMC (pulmonary artery smooth muscle cell) proliferation and resistance to apotopsis [42], relates COPD [39], induces the activation of RAS in the alveolar epithelial cells by tobacco exposure for 5 days a week during 6 months [43] (Figure $8 \mathrm{~B})$. AGE induces the inflammation through the cytokine such as NF kappa B, TNF alpha, IL-1a and IL- $1 \mathrm{~b}[39,41,44]$. RAGE phosphorylates HSP in regard to the persistent of inflammation [34] (Figure 8B). This time, we identified RAGE. This indicates that our proteomic strategy for detecting nuclear phosphoproteins works very well. Though RAGE was usually localized in the cytoplasm of alveolar epithelial type 1 cells and alveolar macrophage in previous reports, RAGE was also observed in the nucleus. Moreover, RAGE was identified as nuclear phosphoproteins, therefore, this result reflects the nuclear localization. In our experiments, RAGE was expressed in the cytoplasm of the control group; on the other hand, RAGE was expressed in both the cytoplasm and the nucleus of the tobacco group. In the previous report, RAGE was highly expressed in the nucleus of the cerebral cortical cells one day after the subarachnoid hemorrhage [45]. Our result showed that a part of RAGE was phosphorylated and moved into the nucleus in the alveolar epithelial cells and the alveolar macrophages in response to the tobacco exposure. These indicate that RAGE was phosphorylated and moved into the nucleus in response to the stress signaling including inflammation by the tobacco exposure (Figure 8B).

On the other hand, SIK2 was decreased by the tobacco exposure. In the neuron after brain ischemia, the glutamic acid bind to NMDA type glutamate receptor and this induces calcium influx. At the same time, SIK2 is degraded and this activates the signaling of the ischemic tolerance [46]. Usually SIK2 phosphorylates TORC and suppress 
Citation: Niimori-Kita K, Nakamura F, Koizumi D, Niimori D (2016) Nuclear Phosphoproteomics Features the Novel Smoking Markers in Mouse Lung Tissue Following Subacute Phase Exposure to Tobacco Smoke. J Bioanal Biomed 8: 009-016. doi:10.4172/1948-593X.1000146
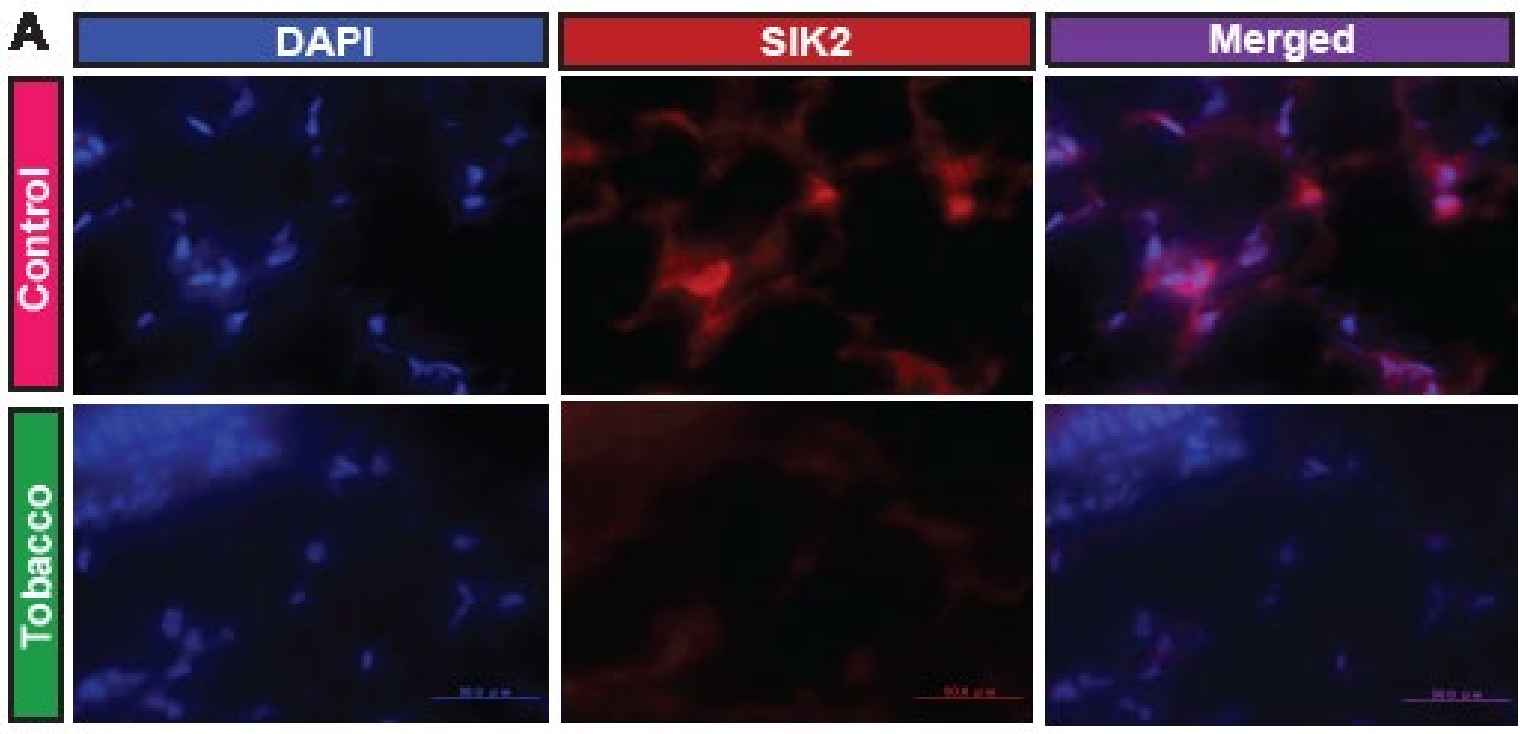

$\mathbf{B}$
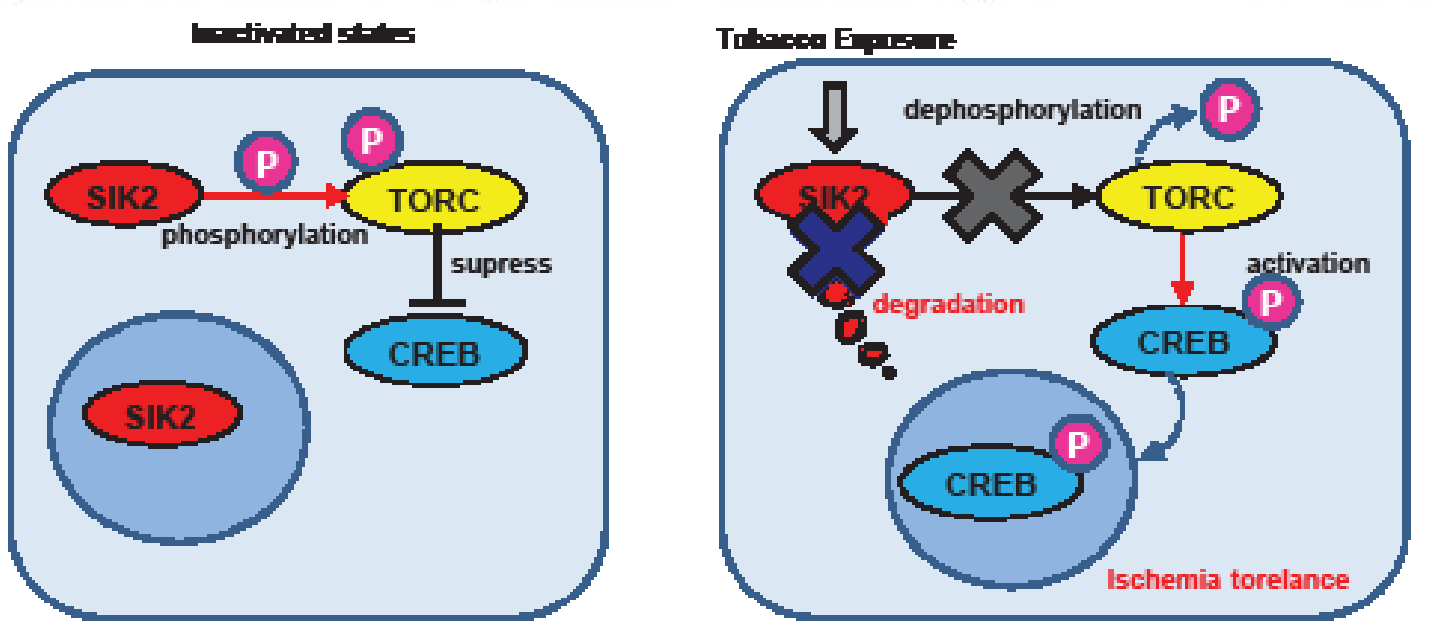

Figure 7: Panel A: Micrographs of the immunofluorescently stained lung tissues obtained from control and mice exposed to tobacco smoke for one month. Panel A shows micrographs of DAPI staining (left), SIK2 staining (center), and the merged image (right; magnification 400X). Panel B: Proposed SIK2 signaling pathways and associated proteins identified as being activated by tobacco smoke exposure. Left panel shows the inactivated states, and right shows the tobacco exposured states.

the TORC-CREB pathway (Figure 7B). Under the ischemia, SIK2 (Thr484) is phosphorylated by CAMK and phosphorylated SIK2 is degraded. Because TORC is not phosphorylated by SIK2, TORC is dephosphorylated. Dephosphorylated TORC phosphorylates CREB, and phosphorylated CREB entered into the nucleus. Finally phosphorylated CREB promotes the transcription of molecules controlling the neural protection and the ischemic tolerance [46,47]. This time, SIK2 was decreased in the lung tissue by the tobacco exposure. In previous, the nitrous oxide emissions of smokers are decreased, and whose blood vessels shrink by the tobacco exposure [7]. Moreover, an intravenous injection of nicotine against rats promotes the phosphorylation of CREB (Serin133) in the adrenal medulla [48]. We observed that SIK2 was expressed in the cytoplasm and the nucleus of the control lung tissues, however, SIK2 was decreased by the tobacco exposure. We thought that SIK2 was decreased in response to the ischemia and nicotine response by the tobacco exposure (Figure 7B).

In conclusion, here, in order to investigate the effect of tobacco smoke exposure in the sub-acute phase, we analyzed nuclear phosphoproteins extracted from murine lung tissue after exposed of mice to tobacco smoke for one month. We found that most of the proteins detected by the present proteomic study are associated with signaling pathways related response, process, activity, regulation and complex, as indicated in (Figure 6). Because smoking is a risk factor of lung diseases including COPD and lung cancer, continuous study of the molecular functions of RAGE and SIK2 will enable us to apply the molecules as potential disease risk markers to prevent these diseases. Furthermore, if the identified molecules are detected in human blood in the future, we will be able to predict the smoking risk easily. However, at the moment, we cannot detect these molecules in human blood, which means insufficiency as risk markers. Still, there are some problems to be solved. Next, we will elucidate signal networks of the peripheral molecules of RAGE and SIK, and target phosphorylated proteins in blood, leading to detection of more practical disease risk markers.

And, not only tobacco smoke, but also diesel exhaust gas has recently attracted our attention as a cause of pollution and cancers. Both tobacco smoke and diesel exhaust gas have toxic substances such 
Citation: Niimori-Kita K, Nakamura F, Koizumi D, Niimori D (2016) Nuclear Phosphoproteomics Features the Novel Smoking Markers in Mouse Lung Tissue Following Subacute Phase Exposure to Tobacco Smoke. J Bioanal Biomed 8: 009-016. doi:10.4172/1948-593X.1000146
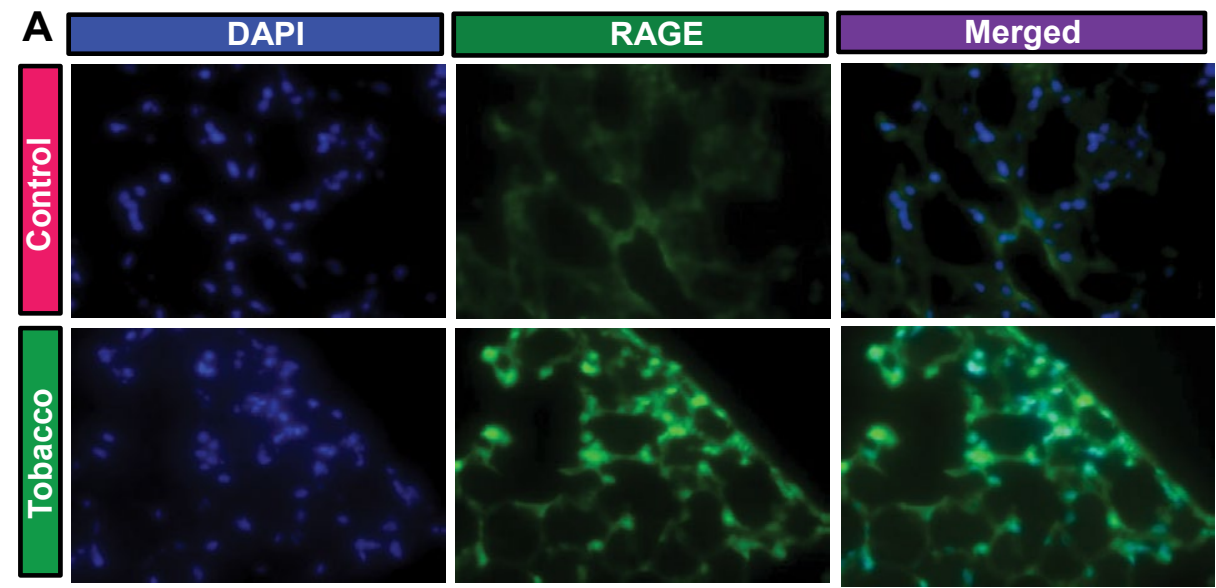

B

\section{Previous Reports}

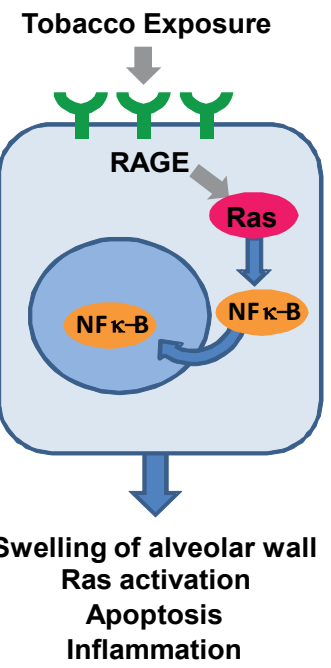

Our Results

Tobacco Exposure

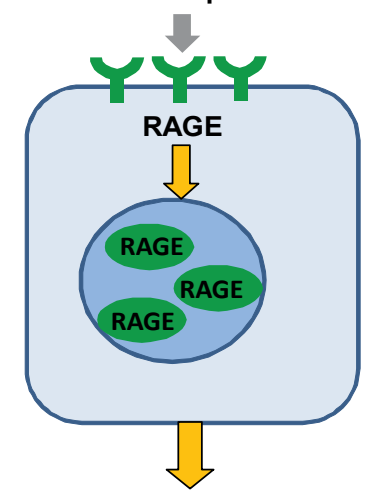

Swelling of alveolar wall

Inflammation

Nuclear localization

Figure 8: Panel A: Micrographs of the immunofluorescently stained lung tissues obtained from control and mice exposed to tobacco smoke for one month Panel A shows micrographs of DAPI staining (left), RAGE staining (center), and the merged image (right; magnification 400X). Panel B: Proposed RAGE signaling pathways and associated proteins identified as being activated by tobacco smoke exposure in previous reports and this study.

as nitrogen oxides and doxin-like chemicals in common. Therefore, we think that the smoking markers identified this time may be applied for markers of diseases caused by diesel exhaust gas. In addition, if there are shared common mechanisms to disease, our experimental strategy may clarify the functional molecules.

\section{Acknowledgement}

\section{General}

Members of Department of Pathology and Experimental Medicine gave us valuable support. Hayato Muroya prepared the smooth research environment Takayoshi Kawaguchi gave us useful comments. Fumihiko Usui, Yasuhiko Bando, Sayaka Mikami did the technical support of mass spectrometry. Noriyuki Nagahara supported our research.

\section{Funding}

This work was supported in part by a grant from the Smoking Research Foundation, by Grants-in-Aid for Young Scientists (Research Project Number: 23790914) and by Shibasaburo Program from Kumamoto University.

The funding source had no role in study design and concept; data collection, analysis, and interpretation; and manuscript preparation and submission.

\section{Specific contributions of each author}

Kanako Niimori-Kita designed experiments Kanako Niimori-Kita, Fumiko Nakamura, Daikai Koizumi and Daisuke Niimori wrote the manuscript Kanako Niimori-Kita, Fumiko Nakamura, Daikai Koizumi and Daisuke Niimori performed the experiments. Kanako Niimori-Kita and Daisuke Niimori performed the in silico analyses. The nuclear phosphoprotein analysis was developed by Kanako NiimoriKita. "Kanako Niimori-Kita and Fumiko Nakamura contributed to this work equally".

\section{References}

1. Hylkema MN, Sterk PJ, de Boer WI, Postma DS (2007) Tobacco use in relation to COPD and asthma. Eur Respir J 29: 438-445.

2. Taylor JD (2010) COPD and the response of the lung to tobacco smoke exposure. Pulm Pharmacol Ther 23: 376-383.

3. Ward SA, Casaburi $R$ (2001) $21^{\text {st }}$ century perspective on chronic obstructive pulmonary disease. Respiration 68: 557-561.

4. Powell HA, Iyen Omofoman B, Baldwin DR, Hubbard RB, Tata LJ (2013) Chronic obstructive pulmonary disease and risk of lung cancer: the importance of smoking and timing of diagnosis. J Thorac Oncol 8: 6-11.

5. Adcock IM, Caramori G, Barnes PJ (2011) Chronic obstructive pulmonary disease and lung cancer: new molecular insights. Respiration 81: 265-284. 
Citation: Niimori-Kita K, Nakamura F, Koizumi D, Niimori D (2016) Nuclear Phosphoproteomics Features the Novel Smoking Markers in Mouse Lung Tissue Following Subacute Phase Exposure to Tobacco Smoke. J Bioanal Biomed 8: 009-016. doi:10.4172/1948-593X.1000146

6. Stinn W, Buettner A, Weiler $\mathrm{H}$, Friedrichs B, Luetjen S, et al (2013) Lung inflammatory effects, tumorigenesis, and emphysema development in a longterm inhalation study with cigarette mainstream smoke in mice. Toxicol Sci 131: 596-611.

7. Churg A, Cosio M, Wright JL (2008) Mechanisms of cigarette smoke-induced COPD: insights from animal models. Am J Physiol Lung Cell Mol Physiol 294 L612-631.

8. Van Der Vaart H, Postma DS, Timens W, Ten Hacken NH (2004) Acute effects of cigarette smoke on inflammation and oxidative stress: a review. Thorax 59: 713-721.

9. Churg A, Zay K, Shay S, Xie C, Shapiro SD, et al. (2002) Acute cigarette smoke-induced connective tissue breakdown requires both neutrophils and macrophage metalloelastase in mice. Am J Respir Cell Mol Biol 27: 368-374.

10. Sopori M (2002) Effects of cigarette smoke on the immune system. Nat Rev Immunol 2: 372-377.

11. Chung KF, Adcock IM (2008) Multifaceted mechanisms in COPD: inflammation, immunity, and tissue repair and destruction. Eur Respir J 31: 1334-1356.

12. Yao H, Rahman I (2011) Current concepts on oxidative / carbonyl stress inflammation and epigenetics in pathogenesis of chronic obstructive pulmonary disease. Toxicol Appl Pharmacol 254: 72-85.

13. Barreiro E, Del Puerto Nevado L, Puig-Vilanova E, Pérez-Rial S, Sánchez F, et al. (2012) Cigarette smoke-induced oxidative stress in skeletal muscles of mice. Respir Physiol Neurobiol 182: 9-17.

14. Hecht SS (1999) Tobacco smoke carcinogens and lung cancer. J Natl Cancer Inst 91: 1194-1210.

15. Hecht SS (2012) Lung carcinogenesis by tobacco smoke. Int J Cancer 131 2724-2732.

16. Osada H, Takahashi T (2002) Genetic alterations of multiple tumor suppressors and oncogenes in the carcinogenesis and progression of lung cancer. Oncogene 21: 7421-7434.

17. Witschi H, Espiritu I, Dance ST, Miller MS (2002) A mouse lung tumor model of tobacco smoke carcinogenesis. Toxicol Sci 68: 322-330.

18. López-Boado YS, Li JU, Clayton CL, Wright JL, Churg A (2010) Modification of the rat airway explant transcriptome by cigarette smoke. Inhal Toxicol 22: 234-244

19. Brody JS (2012) Transcriptome alterations induced by cigarette smoke. Int J Cancer 131: 2754-2762.

20. Marshall H (2012) Genetic and epigenetic factors in development of lung cancer. Lancet Oncol 13: 1188

21. Catassi A, Servent D, Paleari L, Cesario A, Russo P (2008) Multiple roles of nicotine on cell proliferation and inhibition of apoptosis: implications on lung carcinogenesis. Mutat Res 659: 221-231.

22. Moerloose KB, Pauwels RA, Joos GF (2005) Short-term cigarette smoke exposure enhances allergic airway inflammation in mice. Am J Respir Crit Care Med 172: 168-172

23. Arnson $Y$, Shoenfeld $Y$, Amital $H$ (2010) Effects of tobacco smoke on immunity, inflammation and autoimmunity. J Autoimmun 34: J258-265.

24. Wright SC, Zhong J, Zheng H, Larrick JW (1993) Nicotine inhibition of apoptosis suggests a role in tumor promotion. FASEB J 7: 1045-1051.

25. Gebel S, Gerstmayer B, Bosio A, Haussmann HJ, Van Miert E, et al (2004) Gene expression profiling in respiratory tissues from rats exposed to mainstream cigarette smoke. Carcinogenesis 25: 169-178.

26. De Flora S, Balansky R, D'Agostini F, Cartiglia C, Longobardi M, et al. (2012) Smoke-induced microRNA and related proteome alterations. Modulation by chemopreventive agents. Int J Cancer 131: 2763-2773.

27. Niimori-Kita K, Ogino K, Mikami S, Kudoh S, Koizumi D, et al. (2014) Identification of nuclear phosphoproteins as novel tobacco markers in mouse lung tissue following short-term exposure to tobacco smoke. FEBS Open Bio 4: 746-754.

28. Nemmar A, Raza H, Subramaniyan D, John A, Elwasila M, et al. (2012) Evaluation of the pulmonary effects of short-term nose-only cigarette smoke exposure in mice. Exp Biol Med (Maywood) 237: 1449-1456.
29. Xue L, Wang WH, lliuk A, Hu L, Galan JA, et al. (2012) Sensitive kinase assay linked with phosphoproteomics for identifying direct kinase substrates. Proc Natl Acad Sci U S A 109: 5615-5620.

30. Reynolds PR, Kasteler SD, Schmitt RE, Hoidal JR (2011) Receptor for advanced glycation end-products signals through Ras during tobacco smokeinduced pulmonary inflammation. Am J Respir Cell Mol Biol 45: 411-418.

31. Zhang SP, Wu YW, Wu ZZ, Liu HY, Nie JH, et al. (2009) Up-regulation of RAGE and S100A6 in rats exposed to cigarette smoke. Environ Toxicol Pharmacol 28 : 259-264.

32. Schmid RP, Wangensteen D, Hoidal J, Gosnell B, Niewoehner D (1985) Effects of elastase and cigarette smoke on alveolar epithelial permeability. J App Physiol (1985) 59: 96-100.

33. Bolton SJ, Pinnion K, Oreffo V, Foster M, Pinkerton KE (2009) Characterisation of the proximal airway squamous metaplasia induced by chronic tobacco smoke exposure in spontaneously hypertensive rats. Respir Res 10: 118.

34. Carter CA, Misra M, Maronpot RR (2012) Tracheal Morphologic and Protein Alterations FollowingShort-Term Cigarette Mainstream Smoke Exposure to Rats. J Toxicol Pathol 25: 201-207.

35. Valença SS, da Hora K, Castro P, Moraes VG, Carvalho L, et al. (2004) Emphysema and metalloelastase expression in mouse lung induced by cigarette smoke. Toxicol Pathol 32: 351-356.

36. Zhang SP, Wu YW, Wu ZZ, Liu HY, Nie JH, et al. (2009) Up-regulation of RAGE and $\mathrm{S} 100 \mathrm{~A} 6$ in rats exposed to cigarette smoke. Environ Toxicol Pharmacol 28 259-264.

37. Zhang S, Xu N, Nie J, Dong L, Li J, et al. (2008) Proteomic alteration in lung tissue of rats exposed to cigarette smoke. Toxicol Lett 178: 191-196.

38. Verma N, Pink M, Rettenmeier AW, Schmitz-Spanke S (2012) Review on proteomic analyses of benzo[a]pyrene toxicity. Proteomics 12: 1731-1755.

39. Robinson AB, Stogsdill JA, Lewis JB, Wood TT, Reynolds PR (2012) RAGE and tobacco smoke: insights into modeling chronic obstructive pulmonary disease. frontiers in PHYSIOLOGY REVIEW ARTICLE 3: 301.

40. Robinson AB, Johnson KD, Bennion BG, Reynolds PR (2012) RAGE signaling by alveolar macrophages influences tobacco smoke-induced inflammation. Am J Physiol Lung Cell Mol Physiol 302: L1192-1199.

41. Wang G, Han D, Zhang Y, Xie X, Wu Y, et al. (2013) A novel hypothesis: up-regulation of HO-1 by activation of PPAR inhibits HMGB1-RAGE signaling pathway and ameliorates the development of ALI/ARDS. J Thorac Dis 5: 706710.

42. Meloche J, Courchesne A, Barrier M, Carter S, Bisserier M, et al. (2013) Critica role for the advanced glycation end-products receptor in pulmonary arterial hypertension etiology. J Am Heart Assoc 2: e005157.

43. Reynolds PR, Kasteller S, Cosio MG, Sturrock A, Huecksteadt TP, et al. (2008) RAGE: developmental expression and positive feedback regulation by Egr-1 during cigarette smoke exposure in pulmonary epithelial cells. Am J Physio Lung Cell Mol Physiol 294: 1094-1101.

44. Reynolds PR, Cosio MG, Hoidal JR (2006) Cigarette smoke-induced Egr1 upregulates proinflammatory cytokines in pulmonary epithelial cells. Am J Respir Cell Mol Biol 35: 314-319.

45. Li H, Wu W, Sun Q, Liu M, Li W, et al. (2014) Expression and cell distribution of receptor for advanced glycation end-products in the rat cortex following experimental subarachnoid hemorrhage. BRAIN RESEARCH Research Report 1543: 315-323.

46. Sasaki T, Takemori H, Yagita Y, Terasaki Y, Uebi T, et al. (2011) SIK2 is key regulator for neuronal survival after ischemia via TORC1-CREB. Neuron 69: 106-119.

47. Mortaz E, Redegeld FA, Sarir H, Karimi K, Raats D, et al. (2008) Cigarette smoke stimulates the production of chemokines in mast cells. $\mathrm{J}$ Leukoc Biol 83: $575-580$

48. Hiremagalur B, Sabban EL (1995) Nicotine elicits changes in expression of adrenal catecholamine biosynthetic enzymes, neuropeptide $Y$ and immediate early genes by injection but not continuous administration. Brain Res Mol Brain Res 32: 109-115. 